\title{
MIT observatory in last-ditch appeal over withdrawal of NSF funding
}

Boston. Astronomers at the Haystack Observatory in Westford, Massachusetts, will later this month make a last-ditch appeal to the US National Science Foundation (NSF) to reverse a decision to cut all funding for the telescope in four years' time.

Despite a recent three-year, \$1.5-million upgrade, the NSF announced in March that it was cutting funding for the observatory, a research facility of the Massachusetts Institute of Technology, by about 10 per cent, down to about $\$ 1.1$ million.

The future looks even grimmer. Although the NSF has pledged continued support for Haystack's group in very long baseline interferometry (VLBI), it plans to "ramp down" support for the 37-metre radiotelescope over the next three years, terminating it completely after fiscal year 1997 .

The decision has left Haystack personnel reeling, particularly as the renovations mean that it can now operate at frequencies 10 times higher than originally planned. According to Richard Barvainis, an astronomer at Haystack, the latest improvements make it the largest single-dish telescope in the United States operating at 3 millimetres, and the most sensitive in this wavelength band for studying small-diameter sources such as stars and molecular clouds.

Ironically, the NSF decision has come shortly after its officials praised Haystack staff for doing "a superb technical job" with the upgrade. "They not only paid for this work, they also pushed us in this direction," says Joseph Salah, director of the facility. "Now they won't give us a chance to show what the revamped telescope can do."

But the cutbacks were not entirely unexpected. Last year the NSF commissioned a report from a panel led by Joseph Taylor of Princeton University, asking it to evaluate five universitybased observatories, concentrating on millimetre-wavelength radio astronomy. Haystack was rated last, even though the VLBI group was praised, and the recent improvements described as "a substantial technical achievement".

Part of the problem is that Haystack is 30 years old and located close to sea level on the East Coast. "There are limitations as to what you can do with an older telescope in this location," Salah admits. "We're not on top of Mauna Kea."

Yet he and his colleagues also believe that the timing of the NSF review put them at a serious disadvantage, as they had just finished engineering tests after the upgrade, and had no new scientific results to show.

Haystack scientists are not yet throwing in the towel. They see their three-year budget proposal, due at NSF by the end of this

\section{Maths teachers 'would welcome checks'}

Washington. University mathematics departments should take stronger steps to evaluate the quality of teaching by faculty members, and reform pay and promotion structures in a way that rewards good teaching, according to the main US mathematicians' professional societies.

An investigation by the Joint Policy Board for Mathematics - a joint forum of the American Mathematical Society, the Mathematical Association of America and the Society for Industrial and Applied Mathematics - found that, contrary to popular belief, university mathematicians would welcome such changes.

Richard Herman of the University of Maryland, the chairman of the board, says that mathematics departments in public universities should reform themselves to acknowledge the importance of teaching before impatient state governments force them to do so. Herman called on mathematics departments to "think of this as an opportunity" for constructive reform.

The board visited 26 mathematics de- partments and surveyed 2,000 faculty members, and found widespread dissatisfaction about the lack of rewards for teaching. Its report, Recognition and Rewards in the Mathematical Sciences, falls short of recommending that teaching should be rewarded on a par with research, which was by far the predominant performance measure in the departments visited.

In a carefully worded passage, it says that "research should, in general, continue to have a maximal status in the rewards structure". But it calls for a "broader and more flexible" structure that also takes into account teaching, interdisciplinary research and other work in giving promotions and pay increases.

But the report finds deep scepticism about the most widely used means of teacher evaluation, namely the student questionnaire. Mathematics lecturers, it appears, are fed up taking the rap when such surveys place them in a bad light next to their counterparts teaching arts and social science subjects.

Colin Macilwain

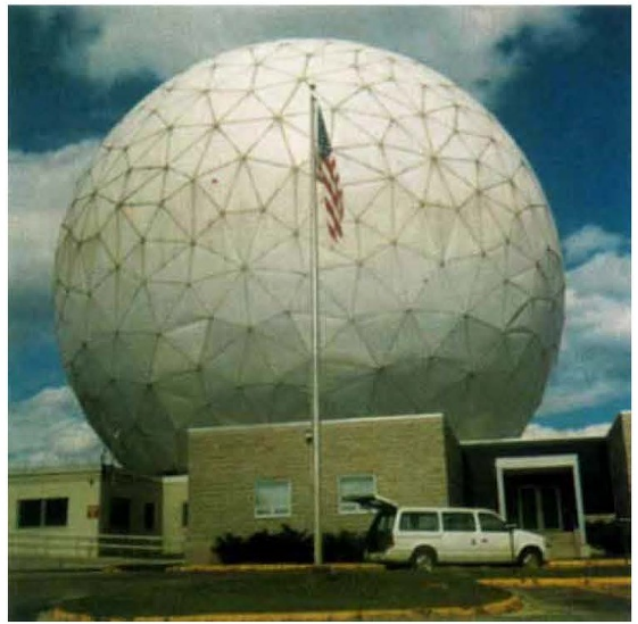

Haystack awaits its fate.

month, as a chance for an appeal. Ways of trimming costs and streamlining operations will be presented. (Jobs have already been lost as a result of the budget cuts, and additional redundancies seem inevitable.)

"We want to emphasize the scientific results obtained since the upgrade and our plans for exploiting the new capability in the future," Salah explains.

Philip Myers, an astronomer at the Harvard-Smithsonian Center for Astrophysics (CFA) and a long-time subscriber at Haystack, believes the telescope can play a "useful role in helping astronomers to solve a long-standing puzzle - how stars form. Most of our understanding of this process is based on inferences rather than direct observations. Haystack has the combination of spatial and spectral resolution needed for the job."

Salah's strategy is to "let the science speak for itself. If we can't make a good scientific case, so be it." He is determined to keep the observatory open, no matter what NSF decides three years from now. The Air Force and NASA already contribute nearly $\$ 5$ million of the \$6-million annual budget, and those sources seem secure for now.

But Hugh van Horn, NSF's astronomy director, is not too encouraging. "This is not a criticism of the science being conducted at Haystack," he says. "The problem is one of limited resources. We need to shift some of those resources to maximize benefits to US astronomy as a whole."

In principle, notes Patrick Thaddeus of the CFA, the concept of pruning commitments is widely accepted. "These days, that's how science is done," he says. "Of course, everyone accepts this idea until they're told that their facility is the one facing the axe."

Steve Nadis 